\title{
HAUSDORFF DIMENSION OF DERANGED CANTOR SET WITHOUT SOME BOUNDEDNESS CONDITION
}

\author{
IN-SOO BAEK
}

\begin{abstract}
A deranged Cantor set(without the uniform boundedness condition away from zero of contraction ratios) whose weak local dimensions for all points coincide has its Hausdorff dimension of the same value of weak local dimension. We will show it using an energy theory instead of Frostman's density lemma which was used for the case of the deranged Cantor set with the uniform boundedness condition of contraction ratios. In the end, we will give an example of such a deranged Cantor set.
\end{abstract}

\section{Introduction}

Under the bounded geometry of contraction ratios of perturbed Cantor sets, we ([1]) studied their Hausdorff and packing dimensions. We ([2]) also investigated the Hausdorff dimension and the packing dimension of a certain deranged Cantor set whose contraction ratios are uniformly bounded. Recently we ([3]) used an energy theory related to Hausdorff dimension to give a lower bound for a perturbed Cantor set whose contraction ratios are not necessarily uniformly bounded away from zero.

In this paper, we also apply the energy theory to some special deranged Cantor sets to find their Hausdorff dimensions. We only consider a particular deranged Cantor set whose lower and upper Cantor local dimensions ([2]) coincide.

\section{Preliminaries}

We recall the definition of the deranged Cantor set ([2]). Let $I_{\phi}=$ $[0,1]$. Then we obtain the left subinterval $I_{\tau, 1}$ and the right subinterval

Received May 21, 2003.

2000 Mathematics Subject Classification: 28A78.

Key words and phrases: Hausdorff dimension, Cantor set, weak local dimension. 
$I_{\tau, 2}$ of $I_{\tau}$ by deleting the middle open subinterval of $I_{\tau}$ inductively for each $\tau \in\{1,2\}^{n}$, where $n=0,1,2, \cdots$. Consider $E_{n}=\cup_{\tau \in\{1,2\}^{n}} I_{\tau}$. Then $\left\{E_{n}\right\}$ is a decreasing sequence of closed sets. For each $n$, we put $\left|I_{\tau, 1}\right| /\left|I_{\tau}\right|=c_{\tau, 1}$ and $\left|I_{\tau, 2}\right| /\left|I_{\tau}\right|=c_{\tau, 2}$ for all $\tau \in\{1,2\}^{n}$,where $|I|$ denotes the diameter of $I$. We call $F=\bigcap_{n=0}^{\infty} E_{n}$ a deranged Cantor set.

We note that if $y \in F$, then there is $\sigma \in\{1,2\}^{N}$ such that $\bigcap_{k=0}^{\infty} I_{\sigma \mid k}=$ $\{y\}$ (Here $\sigma \mid k=i_{1}, i_{2}, \cdots, i_{k}$ where $\sigma=i_{1}, i_{2}, \cdots, i_{k}, i_{k+1}, \cdots$ ). Hereafter, we use $\sigma \in\{1,2\}^{N}$ and $y \in F$ as the same identity freely.

We ([2]) recall the local Hausdorff dimension $f(\sigma)$ of $\sigma$ in $F$

$$
f(\sigma)=\inf \left\{s>0: h^{s}(\sigma)=0\right\}=\sup \left\{s>0: h^{s}(\sigma)=\infty\right\}
$$

where the $s$-dimensional local Hausdorff measure

$$
h^{s}(\sigma)=\liminf _{k \rightarrow \infty}\left(c_{1}^{s}+c_{2}^{s}\right)\left(c_{\sigma \mid 1,1}^{s}+c_{\sigma \mid 1,2}^{s}\right)\left(c_{\sigma \mid 2,1}^{s}+c_{\sigma \mid 2,2}^{s}\right) \cdots\left(c_{\sigma \mid k, 1}^{s}+c_{\sigma \mid k, 2}^{s}\right),
$$

and dually the local packing dimension $g(\sigma)$ of $\sigma$ in $F$

$$
g(\sigma)=\inf \left\{s>0: q^{s}(\sigma)=0\right\}=\sup \left\{s>0: q^{s}(\sigma)=\infty\right\}
$$

where the $s$-dimensional local packing measure

$q^{s}(\sigma)=\limsup _{k \rightarrow \infty}\left(c_{1}^{s}+c_{2}^{s}\right)\left(c_{\sigma \mid 1,1}^{s}+c_{\sigma \mid 1,2}^{s}\right)\left(c_{\sigma \mid 2,1}^{s}+c_{\sigma \mid 2,2}^{s}\right) \cdots\left(c_{\sigma \mid k, 1}^{s}+c_{\sigma \mid k, 2}^{s}\right)$.

We recall the $s$-dimensional Hausdorff measure of $F$ :

$$
H^{s}(F)=\lim _{\delta \rightarrow 0} H_{\delta}^{s}(F),
$$

where $H_{\delta}^{s}(F)=\inf \left\{\sum_{n=1}^{\infty}\left|U_{n}\right|^{s}:\left\{U_{n}\right\}_{n=1}^{\infty}\right.$ is a $\delta$-cover of $\left.F\right\}$, and the Hausdorff dimension of $F$ :

$\operatorname{dim}_{H}(F)=\sup \left\{s>0: H^{s}(F)=\infty\right\}\left(=\inf \left\{s>0: H^{s}(F)=0\right\}\right)([4])$.

Also we recall the $s$-dimensional packing measure of $F$ :

$$
p^{s}(F)=\inf \left\{\sum_{n=1}^{\infty} P^{s}\left(F_{n}\right): \bigcup_{n=1}^{\infty} F_{n}=F\right\},
$$

where $P^{s}(E)=\lim _{\delta \rightarrow 0} P_{\delta}^{s}(E)$ and $P_{\delta}^{s}(E)=\sup \left\{\sum_{n=1}^{\infty}\left|U_{n}\right|^{s}:\left\{U_{n}\right\}\right.$ is a $\delta$-packing of $E\}$, and the packing dimension of $F$ :

$$
\operatorname{dim}_{p}(F)=\sup \left\{s>0: p^{s}(F)=\infty\right\}\left(=\inf \left\{s>0: p^{s}(F)=0\right\}\right)([4]) .
$$

We note that a deranged Cantor set satisfying $c_{\tau, 1}=a_{n+1}$ and $c_{\tau, 2}=$ $b_{n+1}$ for all $\tau \in\{1,2\}^{n}$, for each $n=0,1,2, \cdots$ is called a perturbed Cantor set ([1]).

We are now ready to study the ratio geometry of the deranged Cantor set. 


\section{Main results}

In this section, $F$ means a deranged Cantor set determined by $\left\{c_{\tau}\right\}$ and $d_{\tau}=1-\left(c_{\tau, 1}+c_{\tau, 2}\right)>d>0$ for all $\tau \in\{1,2\}^{n}$ with $n \in N$ and for some $d>0$.

LEMMA 1. $h^{s}(\sigma)=\infty$ if $s<f(\sigma)$ and $q^{s}(\sigma)=0$ if $s>g(\sigma)$.

Proof. It follows immediately from the definitions of $f$ anf $g$.

From now on, we assume that $F$ satisfies $f(\sigma)=g(\sigma)=s$ for all $\sigma \in\{1,2\}^{N}$ for some real number $s \in[0,1)$. We call a deranged Cantor set $F$ satisfies the uniform boundedness condition of $f$ and $g$ if $F$ satisfies the following two conditions that if $v<f(\sigma)=s$ then there exists $A>0$ such that for all $\sigma \in\{1,2\}^{N} r_{k}^{v}(\sigma)=\left(c_{1}^{v}+c_{2}^{v}\right)\left(c_{\sigma \mid 1,1}^{v}+c_{\sigma \mid 1,2}^{v}\right)\left(c_{\sigma \mid 2,1}^{v}+\right.$ $\left.c_{\sigma \mid 2,2}^{v}\right) \cdots\left(c_{\sigma \mid k, 1}^{v}+c_{\sigma \mid k, 2}^{v}\right) \geq A>0$ for all $k \in N$ and if $u>g(\sigma)=s$ then there exists $B<\infty$ such that for all $\sigma \in\{1,2\}^{N} r_{k}^{u}(\sigma)=\left(c_{1}^{u}+\right.$ $\left.c_{2}^{u}\right)\left(c_{\sigma \mid 1,1}^{u}+c_{\sigma \mid 1,2}^{u}\right)\left(c_{\sigma \mid 2,1}^{u}+c_{\sigma \mid 2,2}^{u}\right) \cdots\left(c_{\sigma \mid k, 1}^{u}+c_{\sigma \mid k, 2}^{u}\right) \leq B<\infty$ for all $k \in N$.

LEMMA 2. If there exists $B<\infty$ such that for all $\sigma \in\{1,2\}^{N}$ $r_{k}^{u}(\sigma)=\left(c_{1}^{u}+c_{2}^{u}\right)\left(c_{\sigma \mid 1,1}^{u}+c_{\sigma \mid 1,2}^{u}\right)\left(c_{\sigma \mid 2,1}^{u}+c_{\sigma \mid 2,2}^{u}\right) \cdots\left(c_{\sigma \mid k, 1}^{u}+c_{\sigma \mid k, 2}^{u}\right) \leq B<\infty$ for all $k \in N$, then $\sum_{\tau \in\{1,2\}^{k}}\left|I_{\tau}\right|^{u} \leq B$ for all $k \in N$.

Proof. It is obvious from the comparison between $\sum_{\tau \in\{1,2\}^{k}}\left|I_{\tau}\right|^{u}$ and $r_{k-1}^{u}(\sigma)$.

THEOREM 3. If $f(\sigma)=g(\sigma)=s$ for all $\sigma \in\{1,2\}^{N}$ with the above uniform boundedness condition of $f$ and $g$, then $\operatorname{dim}_{H}(F)=s$.

Proof. Assume that $d_{\sigma \mid n}=1-\left(c_{\sigma \mid n, 1}+c_{\sigma \mid n, 2}\right)>d>0$ for all $\sigma \in\{1,2\}^{N}$ and all $n \in N$ and for some $d>0$.

We may assume that $s>0$ to show that $\operatorname{dim}_{H}(F) \geq s$. Let $0<t<s$ and $s-t=\epsilon$. Consider $s_{1}=t+\frac{3 \epsilon}{4}, s_{2}=t+\frac{\epsilon}{4}$.

We define a set function $\mu$ by

$$
\mu\left(I_{\tau}\right)=\frac{\left|I_{\tau}\right|^{s_{1}}}{r_{n-1}^{s_{1}}(\tau)}
$$

where $r_{n-1}^{s_{1}}(\tau)=\left(c_{1}^{s_{1}}+c_{2}^{s_{1}}\right)\left(c_{\tau \mid 1,1}^{s_{1}}+c_{\tau \mid 1,2}^{s_{1}}\right)\left(c_{\tau \mid 2,1}^{s_{1}}+c_{\tau \mid 2,2}^{s_{1}}\right) \cdots\left(c_{\tau \mid n-1,1}^{s_{1}}+\right.$ $\left.c_{\tau \mid n-1,2}^{s_{1}}\right)$ for each $\tau \in\{1,2\}^{n}$.

Clearly $\mu\left(I_{\tau}\right)=\mu\left(I_{\tau, 1}\right)+\mu\left(I_{\tau, 2}\right)$ for all $\tau \in\{1,2\}^{n}$ for all $n=$ $0,1,2, \cdots$. Then $\mu$ is extended to a mass distribution on $F$ (see [4, Proposition 1.7]). 
Clearly $\lim \inf r_{n-1}^{s_{1}}(\sigma)=\infty$ from $s_{1}<s=f(\sigma)$ by Lemma 1. Further from the uniform boundedness condition of $f$, there is $A>0$ such that $\mu\left(I_{\tau}\right) \leq \frac{\left|I_{\tau}\right|^{s_{1}}}{A}$ for all $\tau \in\{1,2\}^{n}, n=0,1,2, \cdots$.

From the uniform boundedness condition of $g$, since $s_{1}+\left(s_{1}-s_{2}\right)>$ $s=g(\sigma)$,

$$
r_{n}^{s_{1}+\left(s_{1}-s_{2}\right)}(\sigma)<B<\infty
$$

for all $n$ for some $B<\infty$.

For $x, y \in F, x_{\wedge} y$ denote the interval of $E_{n}$ that both $x$ and $y$ belong to with largest integer $n$.

By slight abuse of notation, we write $I \in E_{k}$ to mean that the interval $I$ is one of the basic intervals $I_{\tau}$ of $E_{k}$, where $\tau \in\{1,2\}^{k}$.

If $x_{\wedge} y \in E_{k}$, then for $\tau \in\{1,2\}^{k}$,

$$
\begin{aligned}
& \iint_{x_{\wedge} y=I_{\tau}}|x-y|^{-t} d \mu(x) d \mu(y) \\
\leq & 2 d^{-t}\left|I_{\tau}\right|^{-t} \mu\left(I_{\tau, 1}\right) \mu\left(I_{\tau, 2}\right) \\
\leq & 2 d^{-t} \frac{\left|I_{\tau}\right|^{2 s_{1}-t}}{A^{2}} .
\end{aligned}
$$

Now we see that $\sum_{\tau \in\{1,2\}^{k}}\left|I_{\tau}\right|^{2 s_{1}-t} \leq\left\{(1-d)^{s_{2}-t}\right\}^{k} B$ for each $k=$ $1,2, \cdots$, noting that $a_{i}^{s_{2}-t}, b_{i}^{s_{2}-t} \leq(1-d)^{s_{2}-t}$ for each $i=1,2, \cdots, k$.

Hence

$$
\begin{aligned}
& \int_{F} \int_{F}|x-y|^{-t} d \mu(x) d \mu(y) \\
= & \sum_{k=0}^{\infty} \sum_{I \in E_{k}} \iint_{x_{\wedge} y=I}|x-y|^{-t} d \mu(x) d \mu(y) \\
\leq & 2 d^{-t} \sum_{k=0}^{\infty} \sum_{\tau \in\{1,2\}^{k}} \frac{\left|I_{\tau}\right|^{2 s_{1}-t}}{A^{2}} \\
\leq & \frac{2 d^{-t} B}{A^{2}} \sum_{k=0}^{\infty}\left\{(1-d)^{s_{2}-t}\right\}^{k}<\infty .
\end{aligned}
$$

Since $\mu$ is a mass distribution on $F, \operatorname{dim}_{H}(F) \geq t$ (see [4, Theorem 4.13 (a)]) for every $t<s$. Hence $\operatorname{dim}_{H}(F) \geq s$.

By the uniform boundedness condition of $g, \lim \sup r_{n}^{u}(\sigma) \leq B$ for all $u>s$ for some $B>0$. From Lemma 2 , liminf $\sum_{\tau \in\{1,2\}^{n}}\left|I_{\tau}\right|^{u} \leq B$ for $u>s$. Noting $c_{\tau} \leq 1-d, \operatorname{dim}_{H}(F) \leq s$.

Noting that for every $\sigma \in\{1,2\}^{N}$ in a perturbed Cantor set $F, f(\sigma)$ is the lower Cantor dimension $\operatorname{dim}_{\underline{C}}(F)([1],[3])$ and $g(\sigma)$ is the upper 
Cantor dimension $\operatorname{dim}_{\bar{C}}(F)([1],[3])$, we easily get the following Corollary.

CoRollary $4([3])$. If $F$ is a perturbed Cantor set such that $\operatorname{dim}_{\underline{C}}(F)$ $=\operatorname{dim}_{\bar{C}}(F)=s$, then $\operatorname{dim}_{H}(F)=s$.

Proof. Clearly a perturbed Cantor set has the uniform boundedness condition of $f$ and $g$.

ExAmPle 5. Easily we can find a deranged Cantor set whose $f(\sigma)=$ $g(\sigma)=s$ for all $\sigma \in\{1,2\}^{N}$ with $c_{1, \tau, 1}=a_{n+2}$ and $c_{1, \tau, 2}=b_{n+2}$ and $c_{2, \tau, 1}=a_{n+2}^{\prime}$ and $c_{2, \tau, 2}=b_{n+2}^{\prime}$ for all $\tau \in\{1,2\}^{n}$, for each $n=0,1,2, \cdots$ and liminf of contraction ratios is 0 . Then clearly it satisfies the uniform boundedness condition of $f$ and $g$ above and its Hausdorff dimension is $s$.

Conjecture 6. Consider a deranged Cantor set whose $f(\sigma)=$ $g(\sigma)=s$ for all $\sigma \in\{1,2\}^{N}$ with $c_{1, \tau, 1}=a_{n+2}$ and $c_{1, \tau, 2}=b_{n+2}$ and $c_{2,1, \tau, 1}=a_{n+3}^{\prime}$ and $c_{2,1, \tau, 2}=b_{n+3}^{\prime}$ and $c_{2,2,1, \tau, 1}=a_{n+4}^{\prime \prime}$ and $c_{2,2,1, \tau, 2}=$ $b_{n+4}^{\prime \prime}, \cdots$, for all $\tau \in\{1,2\}^{n}$, for each $n=0,1,2, \cdots$ and liminf of contraction ratios is 0 . We conjecture that it satisfies the uniform boundedness condition of $f$ and $g$ and its Hausdorff dimension is $s$. Further we conjecture that its packing dimension is $s$ also.

\section{References}

[1] I. S. Baek, Dimensions of the perturbed Cantor set, Real Analysis Exchange 19 (1994), no. 1, 269-273.

[2] Weak local dimension on deranged Cantor sets, Real Analysis Exchange 26 (2001), no. 2, 553-558.

[3] _ Hausdorff dimension of perturbed Cantor sets without some boundedness condition, Acta Math. Hungar. 99 (2003), no. 4, 279-283.

[4] K. J. Falconer, Fractal geometry, John Wiley and Sons, 1990.

Department of Mathematics

Pusan University of Foreign Studies

Pusan 608-738, Korea

E-mail: isbaek@pufs.ac.kr 